\title{
Albert Heim (1849-1937)
}

\section{Frau Elisabeth Heim-von Brasch in Verehrung gewidmet}

Von S. Franks und B. Glaus

\begin{abstract}
«... Man hörte nach einer Weile den alten Tal durch die Vorhalle kommen, kannte bald seine besondere Gangart und den ungleichen Takt seiner Schritte, denn der alte Tal litt an einem Gebrechen von Jugend an: er hatte ein verkürztes Bein und schritt mit diesem auf einer hohen Sohle einher, wie man in Wasenwachs bei Tauwetter hohe Stöckel Schnee unter die Holzschuhe bekam. Gleich darauf kam er in den Vorlesungssaal herein, schritt rasch zum Katheder, zog ein paar lose Blätter aus der Tasche und begann zu sprechen.

Sein Gegenstand: die Erde. Großartig war's, richtig großartig, so wie Oeppi es gar nicht geahnt hatte ...»
\end{abstract}

Arnold Kübler, «Oeppi der Student» (p.22) ${ }^{1}$

«Noch heute, hundert Jahre nach seiner Geburt, ist die Erinnerung an Albert Heim als Mensch und Forscher, nicht nur unter Gelehrten aller Erdteile, sondern bis in alle Volksschichten seiner Heimat lebendig.» Mit diesen Worten eröffnete der Sohn, Arnold Heim, die 1952 erschienene Biographie ${ }^{2}$. 30 Jahre später hat seine Aussage wohl nur mehr bedingte Gültigkeit. Heim Vater und Sohn sind zwar den Leuten vom Fach: Geologen und Ingenieuren, immer noch ein Begriff; darüber hinaus aber ist ihr Andenken wohl weitgehend unserer kurzlebigen Zeit zum Opfer gefallen. Während es von Arnold Heim, trotz ausgezeichneter Quellenlage, noch keine erschöpfende Lebensbeschreibung gibt ${ }^{3}$, erfüllt diejenige Albert Heims wohl grosso modo nach wie vor die Ansprüche einer guten Lebens- und Fachgeschichte. Doch hat ihr Vorteil: der Überblick, auch den Nachteil, daß bedeutsame Einzelaspekte darin ihrer «monographischen» Behandlung entbehren müssen, obwohl sie sie verdienten. Wenn unser Beitrag dazu anregen würde, wäre sein Zweck mehr als erfüllt!

\section{Biographischer Abriß}

Albert Heim wurde am 12. April 1849 in Zürich geboren, als zweites Kind von Johann Konrad Heim aus St. Gallen und der Zürcher Bürgerin Sophie 
geborene Fries ${ }^{4}$. «From his father, a merchant, Heim gained his lifelong interest in mountain scenery, particulary as a result of a walking tour in 1865. From his mother he inherited his talent for drawing, which was a powerful vehicle for describing the complex Alpine geological structures he studied throughout his life.. ${ }^{5}$ Heim besuchte die Industrieschule in Zürich, die er 1866 mit der Matura abschloß. Das Studium begann er an der Universität, um «Naturforscher» zu werden, welchselte dann ans Polytechnikum (jetzt: Eidgenössische Technische Hochschule Zürich, ETHZ) über und diplomierte hier 1869 zum «Fachlehrer in naturwissenschaftlicher Richtung». Als Diplomarbeit hatte ihm sein Mentor Arnold Escher von der Linth (1807-1872), Geologieprofessor der Universität und der ETH, ein Thema aus der Gletschergeologie gegeben. An der Universität Berlin und auf Reisen (Nord- und Ostdeutschland, italienische Vulkane) bildete der junge Akademiker sich weiter; doktorieren wollte er aus Prinzip nicht. «Nach Zürich zurückgekehrt, arbeitete er vor allem in den Hochschulsammlungen, die er mit Gesteinsproben und Fossilien von seinen Reisen bereichert hatte. ${ }^{6} 1871$ habilitierte er sich sowohl am Polytechnikum als auch an der Universität. Als Escher starb, setzte Schulratspräsident Karl Kappeler (1816-1888) die Wahl des knapp 23jährigen zum Nachfolger an der ETH durch; 1874 zog die Universität gleich. Heim prägte bis zu seinem (etwas vorzeitigen) Rücktritt vom Lehramt, 1911, Generationen von Geologen, Naturwissenschaftern und Ingenieuren. Selbst ein so kritischer Geist wie Albert Einstein war von diesem Lehrer beeindruckt. Neben dem Unterricht entfaltete Heim eine ausgedehnte publizistische und Expertentätigkeit.

1874 verlobte er sich mit der kurz zuvor promovierten ersten Schweizer Ärztin Dr. med. Marie Vögtlin aus Aarau (1845-1916) ${ }^{7} ; 1875$ heirateten die beiden. Die ärztliche Praxis an der Hottingerstraße (wo sich heute das Athenäum befindet) blieb das gemeinsame Domizil, während das nach Heims Plänen erbaute «Chalet Hagrose» (Hofstraße 100, ebenfalls im Kreis 7) ab 1883 als Sommerhaus, später aber als Alterswohnsitz diente. Der Ehe entsprossen die Kinder Arnold (1882-1965), der das wissenschaftliche Erbe des Vaters weitertrug, und Helene (1886-1979), die Krankenschwester wurde; ein drittes Kind, Rösli, starb kurz nach der Geburt (1889).

Albert Heim wurde 1881 zum Direktor der Geologischen Sammlungen und zum Vorsteher der naturwissenschaftlichen Abteilung des Polytechnikums (VI B) ernannt. Darüber hinaus versah er die verschiedensten fachlichen und gemeinnützigen Ämter, so das Präsidium der Naturforschenden Gesellschaft Zürich, des Feuerbestattungsvereins und der Zürcher Kinder- 


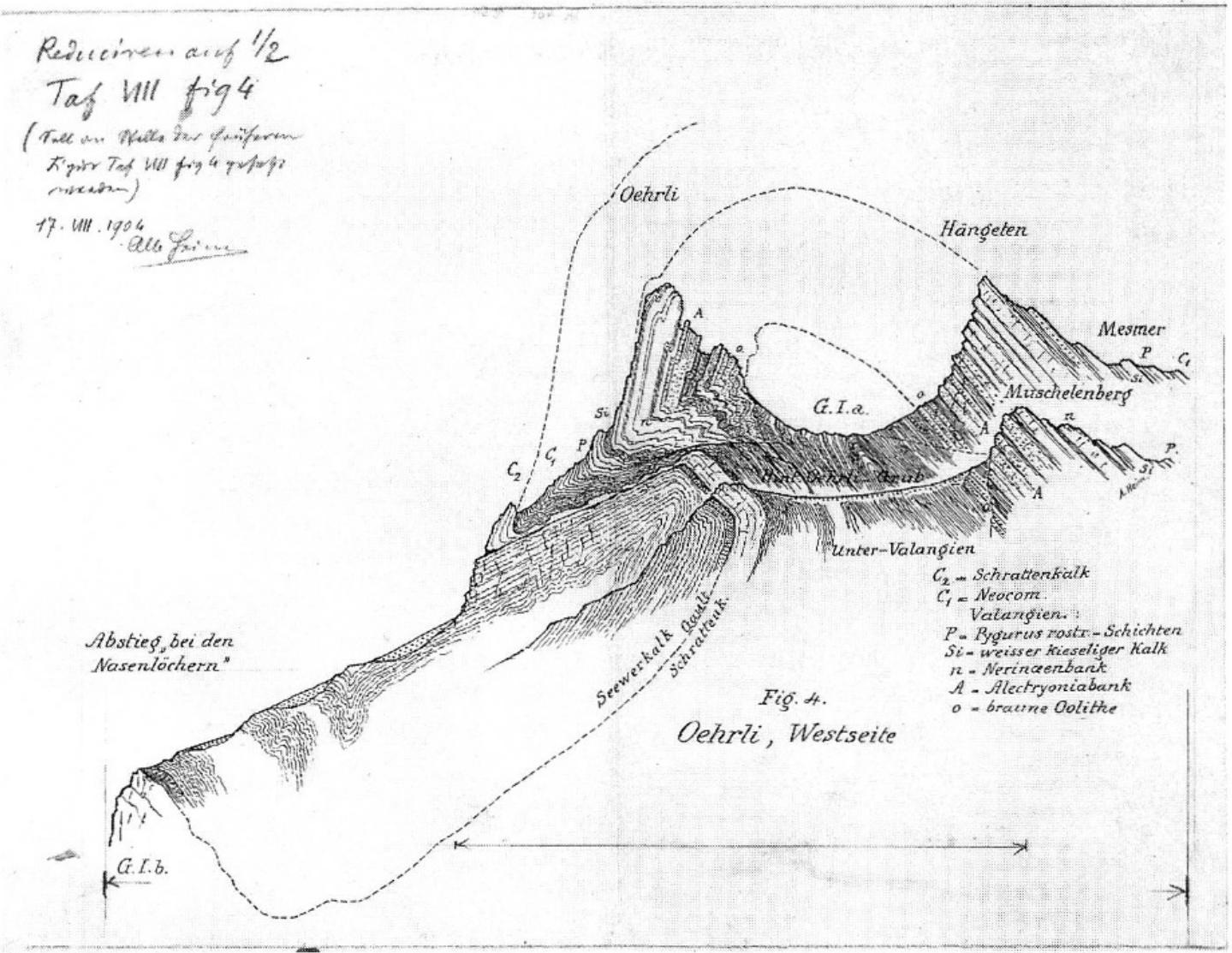

Profilansicht des «Oehrli» im Säntisgebirge Tuschzeichnung von Albert Heim (ETH-Bibliothek)

gärten. 32 Jahre lang präsidierte er die Geologische Kommission zur Landeskartierung, und sozusagen sein Leben lang war er passioniertes Mitglied des Schweizer Alpen-Clubs (SAC) und versierter Kynologe. Heims Renommée im In- wie im Ausland war beträchtlich, wohl an die hundert Ehrungen und Auszeichnungen bezeugen dies.

Auch an Schicksalsschlägen fehlte es der Familie nicht: So zahlte sie jahrelang, allerdings freiwillig, Schulden zurück, die der Zusammenbruch des väterlichen Bankgeschäftes hinterlassen hatte. Nur schwer ertrug die Mutter den Verlust ihres dritten Kindes, und Heim selber verwand nicht leicht den frühen Tod dreier hervorragender Schüler, Alexander Wettstein (1861-1887), Gustav Maillard (1860-1891) und Léon du Pasquier (18641897). Auch der selbständige Weg, den sein Sohn einschlug, als er sich 1911 der Nachfolgekandidatur fürs Professorenamt entzog, enttäuschte den 
Alternden zutiefst. Heim verdankte seine bis ins Alter ungebrochene Schaffenskraft, die ihn zu einem immensen Lebenswerk befähigte, neben reicher Begabung einer soliden, suchtfreien Lebensweise und stählerner Willenskraft. Von einem schweren Unfall blieb dem Kantonsschüler ein verkürztes Bein zurück; der Junge verarbeitete das Unglück, indem er «einen ganzen Winter lang täglich im Zürichsee» badete und sich zum «ausgezeichneten und fast unermüdlichen Bergsteiger» ausbildete ${ }^{8}$. Nicht ohne Grund trägt die SAC-Hütte am Winterstock oberhalb Realp seinen Namen! Es ist bezeichnend für seinen Wagemut, daß Heim auch Spelterinis abenteuerliche Ballonfahrten der 1890er Jahre seinen Zwecken dienstbar machte.

Albert Heims Lebensabend, überschattet vom vorzeitigen Tode der Gattin, war hauptsächlich wissenschaftlichen Studien, fortgesetzter Expertentätigkeit, verschiedener Öffentlichkeitsarbeit sowie seinem kynologischen Steckenpferde gewidmet. Heim starb, umsorgt von seiner Tochter, am 31. August 1937.

Das Werk

«... Der alte Tal war ein Menschenleben lang jenen Stätten nachgereist, da die Erde ihr felsiges Innere den Blicken preisgab. Von Jugend an hatte er keine andere WissensLeidenschaft gehabt. Er war ein Forscher. Er erzählte nicht nur Gewußtes, sondern erzählte Erfahrenes. Frühe Irrtümer waren die Mütter später Einsichten geworden. Er erzählte von den Leiden der Fragen, von den Schmerzen der Zweifel, von den Freuden der Einsicht. Er sprach ruhig, ohne Schärfe, freundlich, ja fast väterlich und blickte aus hellen blauen Augen durch eine dicke Brille nach der Hörerschaft. Sein schneeweißer Bart war lang und wuchs frei, wie's bei manchem Wasenwachser Großvater der Fall war. Ein Kranz weißer langer Haare zog sich um das kahle Gewölbe des Schädels. Man hätte sagen können, daß dessen Rund sich wie ein kahler Steinbuckel aus einer blühenden weißen Wiese erhoben habe. Oeppi hörte ihm ungemein gerne zu. Obendrein war das Vorgetragene ohne Mühe verständlich ...»

«Oeppi der Student» (p.28-29)

«Heim was the first genuin European geological artist; his talent lay in his power to describe accurately the most complex geological structures and to 


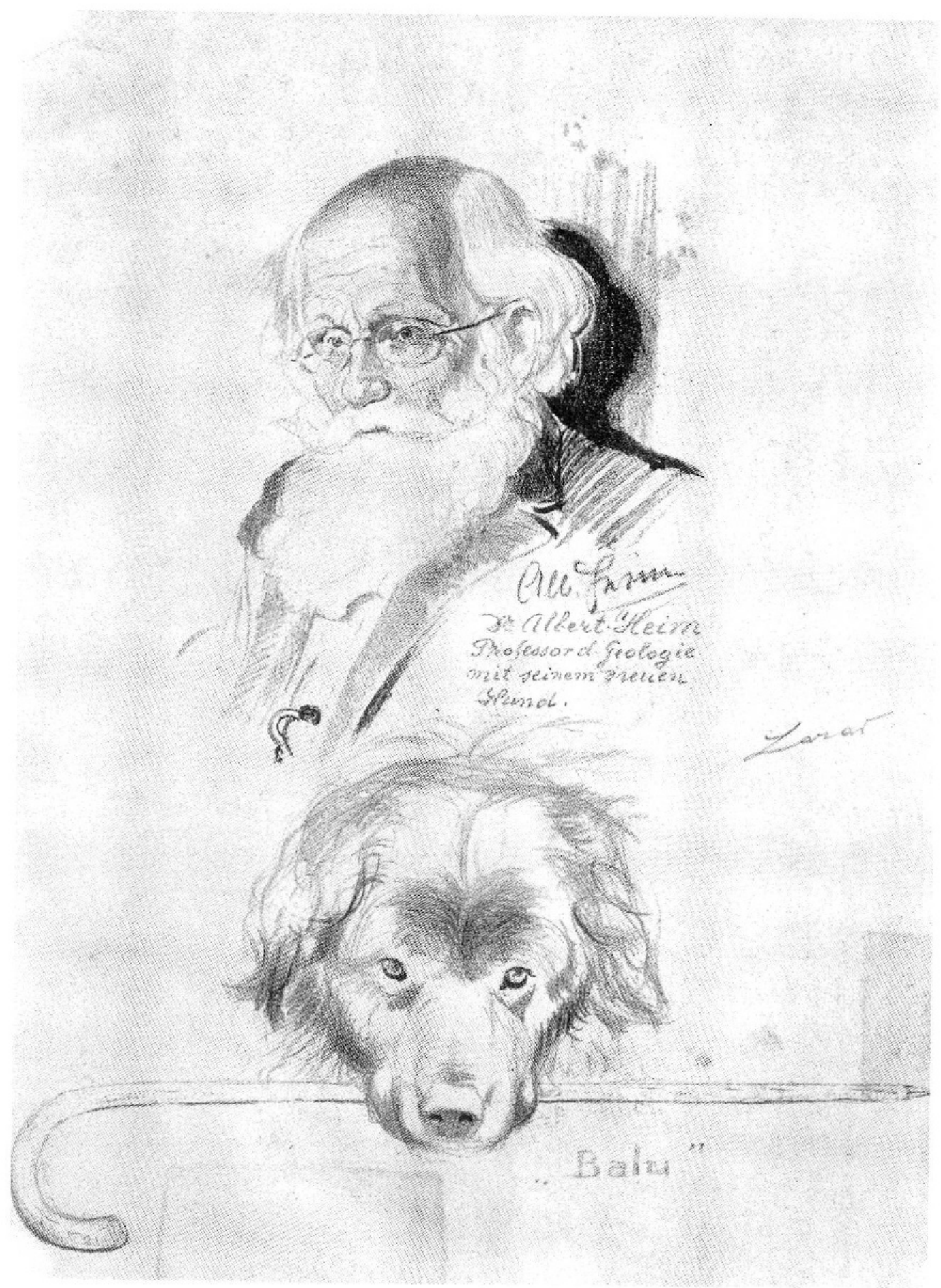

Selbstbildnis mit Hund

(Abdruck einer Zeichnung, ETH-Bibliothek) 
illustrate them with brillant drawings, cross sections and models.» ${ }^{9}$ Der komplizierte Bau der Schweizer Alpen war vor allem durch seinen Vorgänger Arnold Escher von der Linth und den Berner Geologieprofessor Bernhard Studer (1794-1887) in den großen Prinzipien erkannt worden. Die Forschertätigkeit Heims und seiner Schüler bereicherte nicht nur die Alpen-, sondern die gesamte Schweizer Geologie durch unzählige neue Beobachtungen und Detailstudien. Schon um 1840 hatte Arnold Escher in den Glarner Alpen die Dekennatur der Gesteinsschichten richtig erkannt ${ }^{10}$. Im Widerspruch hierzu entwickelte Heim seine spektakuläre These von der «Glarner Doppelfalte». Indessen setzte sich die 1884 erstmals von Marcel Bertrand (1847-1907) vorgetragene und von Eduard Suess (1831-1914), Hans Schardt (1858-1931), Maurice Lugeon (1870-1953) und anderen unterstützte Deckenlehre mehr und mehr durch, bis Heim 1903 sich schließlich ebenfalls zu ihr «bekehrte».

1878 erschien das bahnbrechende Werk «Untersuchungen über den Mechanismus der Gebirgsbildung, im Anschluß an die geologische Monographie der Tödi-Windgällen-Gruppe» (2 Bände und Atlas). Heim legte darin gewissermaßen den Keim für die moderne Strukturgeologie und erkannte Probleme der Gesteinsdeformation, die aber von ihm und seinen Nachfolgern nicht oder nur am Rande weiterverfolgt und erst in den letzten Jahrzehnten systematisch bearbeitet wurden. Dieses Werk erscheint daher heute als eine Art Prototyp manches modernen Lehrbuches der Strukurgeologie ${ }^{11}$. Weitere Forschungsschwerpunkte Heims waren das Säntisgebirge und die Geologie zwischen Reuß und Rhein; diese Gebiete wurden von ihm ebenfalls monographisch und geologisch-kartographisch bearbeitet. Das Ergebnis all dieser Forschungen und derjenigen seiner Schüler faßte er nach beendeter Lehrtätigkeit - zusammen in der «Geologie der Schweiz» (2 Bände, 1916-1922), einem Werk, das heute mitnichten veraltet ist; gilt es doch selbst Geologen von Rang «immer noch als das beste Buch über Schweizer Geologie». Heim war seit 1877 Mitglied der (1860 gegründeten) Schweizerischen Geologischen Kommission (einem Organ der Schweizerischen Naturforschenden Gesellschaft), und 1894-1926 ihr Präsident. Unter seiner Ägide begannen die Aufnahmen der Geologischen Spezialkarten 1:50000 und 1:25000 sowie die «Neue Folge» der dazugehörigen «Beiträge», deren farbgetreue Drucklegung er in all ihren Feinheiten persönlich überwachte.

Ein weiteres Hauptwerk, das «Handbuch der Gletscherkunde» (1885), befaßte sich, anknüpfend an seine Diplomarbeit, mit dem Studium der 


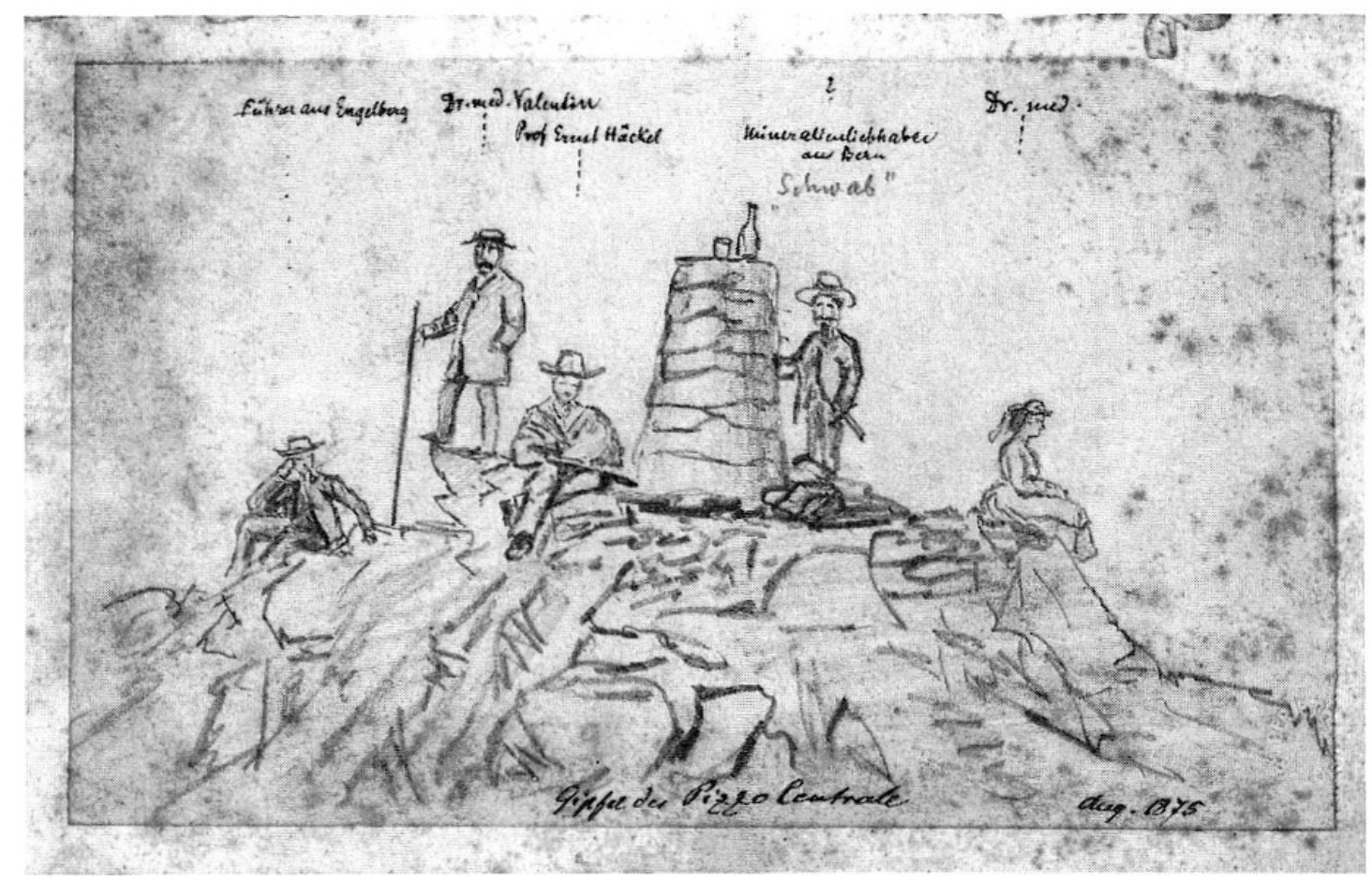

Auf dem Gipfel des Pizzo Centrale, August 1875 (mit Ernst Häckel) Bleistiftskizze von Albert Heim (ETH-Bibliothek)

Gletscherbewegung und zeigte moderne Methoden zur Gletschermessung auf. Unzählige Resultate eigener Forschung sind darin integriert, und zeit seines Lebens ließ das Spezialgebiet der Glazialgeologie Heim nicht los. Noch als 82jähriger publizierte er eine «Geologie des Rheinfalls» (1931). Während 19 Jahren präsidierte er die Schweizer Gletscherkommission, die ihn in der Folge zum Ehrenmitglied ernannte. Seine Korrespondenz mit namhaften Physikern belegt, daß Heim in Fragen der Gletscherforschung wie auch der mechanischen Gesteinsumformung sich bemühte, seine Beobachtungen physikalisch richtig zu untermauern.

Bahnbrechend wirkte Heim schließlich auch in der geologischen Kartographie. Nicht nur schuf er selber prachtvolle geologische Karten der von ihm in langwieriger Feldarbeit kartierten Gebiete. Entgegen der damals üblichen und teilweise wenig befriedigenden Darstellungsart setzte er sich auch mit Verve für vernünftige topographische Kartengestaltung ein (richtige Beleuchtung, sprechende Farbwahl, usw.), in teilweiser Vorwegnahme späterer Forderungen Eduard Imhofs. Heim ist bekannt für seine prachtvollen Alpenpanoramen. Die neue Auflage des Mythenpanoramas, die der 72 jährige veröffentlichte, gilt als sein vollendetstes Werk dieser Art. 
Schon als Knabe übte er sich im Reliefieren und zog damit die Aufmerksamkeit Arnold Eschers auf sich. Zu Lehrzwecken entstanden 1873-1883 mehrere Typenreliefs, die in viele Lehrsammlungen Eingang fanden. Als bedeutendstes «Heim-Relief» gilt das von Carl Meili (1871-1919) unter Heims Leitung ausgeführte Säntis-Relief (1903) ${ }^{12}$.

Albert Heim hielt während seiner fast vier Jahrzehnte dauernden Dozententätigkeit etliche hundert Vorlesungen, Übungen, Seminarien und Exkursionen. Er war ein hervorragender Didaktiker, trug (nach ein paar Stichwörtern) frei vor, ließ an der Tafel zeichnerisch die Alpen entstehen und wieder abtragen, unterstützt von Tafelbildern und von Gesteinsproben, die von Hand zu Hand gingen ${ }^{13}$. Glanzstücke waren die Exkursionen, die Heim mit seinen Studenten, aber auch mit den internationalen Fachgenossen unternahm. Es waren unvergeßliche Erlebnisse, wie viele seiner Schüler bekannten ${ }^{14}$. Heim war nicht, wie viele Geologieprofessoren heute, auf ein Spezialgebiet fixiert; er war in der Eiszeitlandschaft des Kantons Zürich genauso zu Hause wie bei den alten Vulkanen des Hegaus, in den Graniten und Gneisen des Aarmassivs oder den diversen Gesteinsschichten am Walensee. Die von Escher angelegte geologische Lehrsammlung, von Heim mustergültig ausgebaut, galt als die erste ihrer Art in Europa. Sie wurde u. a. in Wien kopiert - hat allerdings am Polytechnikum selber die verschiedenen Umbauten und namentlich die - stark «amuseale» - Fortschrittseuphorie der letzten Jahrzehnte nicht unbeschadet überstanden ...

Heim wurde bald einmal «als Experte in technischen Fragen der angewandten Geologie» beansprucht und erstattete in der Folge einige hundert Berichte, von denen die wenigsten gedruckt vorliegen dürften. Es war dies sein "geologisch-technisches Laboratorium», von dem er für Unterricht und Forschung zehrte ${ }^{15}$. Er verstand es mustergültig, sich auf das Wesentliche zu beschränken, ohne sich in Detailbeschreibungen zu verlieren. Wozu es heute meist mehrere Mitarbeiter samt Ingenieuren, Zeichnern und Sekretärinnen braucht, das erledigte Heim sozusagen im Einmannbetrieb und gegen bescheidenstes Honorar. So begutachtete er Bergstürze, Rutschungen, Bahnlinien, Tunnels, Wasserkraftwerke, Erzvorkommen, Mineralien und Nutzgesteine, Lawinenzüge, Gewässerkorrekturen, Thermen u.a.m. Ein Fazit solcher Tätigkeit ist seine Studie «Bergsturz und Menschenleben» (1932), die noch heute als unübertroffenes Meisterwerk gilt.

Neben all dem betätigte sich Heim aber auch als anerkannter Kynologe. In den 80er Jahren begründete er eine Neufundländer-Zucht, die bald im In- 
und Ausland gefragt war. Ferner nahm er sich unserer Sennenhunde an, unter denen er eine neue Rasse, den «großen Schweizer Sennenhund», entdeckte, und förderte ihre Züchtung und Pflege. Heim veranstaltete mit seinen Hunden unter anderem Wasserdressuren im Zürichsee und verwendete sie als Nutztiere; sie zogen z. B. im Winter Schlitten zum Chalet Hagrose hinauf. Dank seinem Einsatz wurde der Zughund in der schweizerischen Kynologie salonfähig. Heim zu Ehren trägt die prähistorische kynologische Sammlung des Naturhistorischen Museums in Bern den Namen «AlbertHeim-Stiftung», und die «Heim-Plakette» dient noch heute als Auszeichnung erstprämierter Neufundländer- und Sennenhunde.

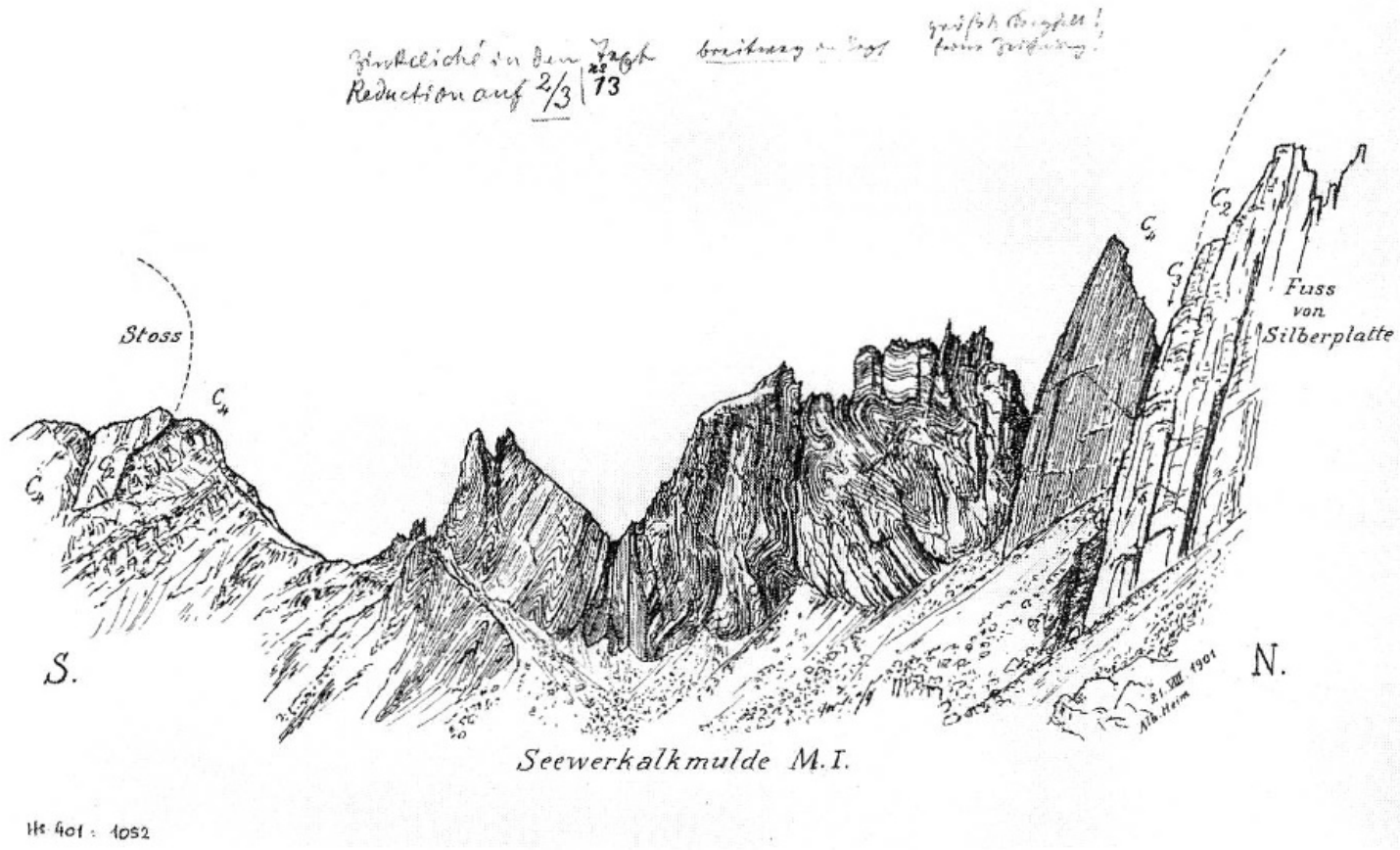

«Seewerkalkmulde» zwischen Stoos und Silberplatte im Säntisgebirge Tuschzeichnung von Albert Heim (ETH-Bibliothek)

Nicht unerwähnt darf der Ethiker Albert Heim bleiben! Selber abstinent geworden, bekämpfte er Alkoholismus und Rauchen in Wort und Schrift, ganz im Einklang mit seiner Frau. Früh setzte er sich für die Feuerbestattung ein, und nicht zuletzt seinem Einfluß ist es zuzuschreiben, daß 1889 das erste Krematorium der Schweiz in Zürich entstand, als drittes in Europa. Er trat für eine rigorose, Achtung für die Frau und Gleichberechtigung heischende Sexualmoral ein und scheute sich nicht, diese Ansichten auch öffentlich vor den Studenten zu vertreten - was nicht überall Anklang fand. 
Als großer Tierliebhaber sprach er sich gegen die Vivisektion aus und kämpfte für den Schutz des Tieres. Im stillen übte er sich, durch unzählige Gaben, als praktischer Philanthrop. Heim wirkte aber auch in den politischen Bereich hinein. Obwohl alles andere als ein sturer Kraftwerkgegner, bezog er den «modernen» Standpunkt des Naturschutzes stets in seine Überlegungen ein. Der Schutz der Landschaft bewog ihn zur Ablehnung einer Stauung des Silsersees (1910), und praktisch ein Leben lang wirkte er für die Erhaltung des Rheinfalls, am nachdrücklichsten wohl mit seinem Aufruf von 1933. 1928 rief die sogenannte Zürcher «Kursaal-Initiative» den Gegner der «Spielbanken» aufs öffentliche Parkett. Der Völkerbund war für Heim ein Instrument der «Siegerstaaten» des Ersten Weltkriegs und als Beeinträchtigung der schweizerischen Neutralität inakzeptabel. Den Beitritt der Schweiz beantwortete er deshalb mit engagierter Anteilnahme am gegnerischen «Volksbund für die Unabhängigkeit der Schweiz».

Albert Heim war kein «Kirchenchrist» im üblichen Sinne des Wortes. Doch wurzelte sein Fühlen und Handeln in einer starken und freien Religiosität. Während seines langen Todeskampfes «rief er oft stundenlang unablässig nach Gott und Christus» ${ }^{16}$. Unter seinen Abschiedsnotizen für die Angehörigen fand sich die Aussage:

«Von einem Berge schaue ich über das weite Land und zur untergehenden Sonne. Die Abendglocken verklingen von allen Seiten. Mir ist, meine Frau lege schweigend ihre Hand in die meine. Mein Leben war Entsagung, mein Leitstern die Pflicht, und doch war es werth, gelebt zu werden. Wie schöne leise Musik ziehen Friede und Vergebung in meine müde Seele ein.» ${ }^{17}$

\section{Der Nachlaß}

Wohl am wirkungsmächtigsten ist das Erbe, das Heim durch Lehre und Forschung in seinen Schülern hinterließ. Doch nicht von diesem unwägbaren Schatz ist hier abschließend kurz zu handeln, sondern von dem uns überkommenen dokumentarischen Nachlaß.

Die Bibliographie des gedruckten Euvre sowie der Reliefs dürfte einigermaßen vollständig sein. Ärgerlicherweise ist sie auf drei verschiedene Zeitschriftenfaszikel verstreut ${ }^{18}$. Mehrere von Heims Monographien sind heute antiquarisch gesuchte Werke. Exemplare Heimscher Reliefs befinden sich in den verschiedensten Schweizer Sammlungen ${ }^{19}$; im Geologischen Institut der ETH Zürich dürften die meisten davon noch vorhanden sein. Heims Arbeitsarchiv, soweit es erhalten ist, befindet sich in den Handschrif- 
ten-Abteilungen der Zentral- und der ETH-Bibliothek Zürich. Die beiden Sammlungen ergänzen sich aufs beste. Die Zentralbibliothek erhielt nach dem Zweiten Weltkrieg größtenteils das von Heims ehemaliger Assistentin Frau Dr. Marie Brockmann-Jerosch (1877-1952) und subsidiär von Arnold und Helene Heim selber geordnete Material, welches die Grundlage für die Biographie geboten hatte. Es dürfte mengenmäßig etwa zwei Drittel des erhaltenen schriftlichen Nachlasses ausmachen. Den wissenschaftlich wohl wertvollsten Teil bilden Feld- und Notizbücher, die sozusagen das A und O des Geologen sind und seine primären Beobachtungen festhalten. Sie dürften hier ziemlich lückenlos vorhanden sein. Auch Heims Unterrichtstätigkeit ist einigermaßen aktenkundig. Eigene Dossiers dokumentieren teils im Manuskript, teils skizzenhaft seine berühmten Vorlesungen «Urgeschichte des Menschen», «Allgemeine Geologie», «Geologie der Schweiz», «Geologie der Gebirge», "Organische Geologie», aber auch kleinere Vorlesungen sowie Exkursionen. Beeindruckend ist der Niederschlag, den Heims reiche Expertentätigkeit gefunden hat; er macht sowohl in der ZB wie in der ETH-Bibliothek fast die Hälfte des jeweiligen Nachlasses aus! Bezüglich Heims Anschauungsmaterial (Zeichnungen, Profile, Aquarelle, Panoramen, Photographien) ist die ETH-Bibliothek besser dotiert. Hier finden sich auch etliche Notizhefte, in denen Studenten Heims Vorlesungen festgehalten haben; angesichts der generationenlangen Unterrichtstätigkeit Heims wirkt der archivierte Bestand allerdings bescheiden: Ergänzungen wären infolgedessen sehr willkommen! Biographisches Quellenmaterial (autobiographische Ansätze, Ehrungen, Erinnerungen, familiäre Aufzeichnungen, Gedichte, usw.) sowie Korrespondenzen, Akten zur Kommissionstätigkeit und vom gesellschaftlichen oder privaten Engagement Heims sind in beiden Sammlungen zu finden. Summa summarum dürfte dieser gehaltvolle Nachlaß wohl Stoff für mehrere - fach- wie kulturgeschichtlich interessierte - Arbeiten abgeben. So wäre wohl eine aufgrund der Quellen geschriebene Darstellung von Heims Weg zur Deckenlehre ein geologiegeschichtlich recht nützliches Unternehmen. Auch der in seiner Natur und Menschen schützenden Ethik teilweise sehr modern anmutende Mann wäre einer eingehenden und aktualisierten Würdigung wohl wert. Moderne Pioniere aber waren Marie Heim-Vögtlin und Albert Heim nicht zuletzt in ihrer Verwirklichung einer auf Gleichberechtigung beruhenden Familiengemeinschaft, und zumindest dieses (heute vielleicht noch am Persönlichkeitsschutz scheiternde) Thema könnte, bei guter quellengetreuer Bearbeitung, wohl einen wesentlichen Beitrag zur Soziologie der Moderne leisten! 


\section{Anmerkungen}

1 Morgarten-Verlag, Zürich, 1947. Küblers Oeppi-Romane sind stark autobiographisch. Der Verfasser studierte von 1910 bis 1915 (mit Unterbrechungen) an der Universität Zürich Naturwissenschaften, insbesondere Geologie. Das Fach wurde, wie die Erdwissenschaften überhaupt, in sogenannten «Doppelprofessuren» an Eidgenössischer Technischer Hochschule (ETH) und Universität Zürich von den gleichen Lehrern betreut. Die geologischen Vorlesungen fanden zu «Oeppis» Zeiten noch im Semperbau der ETH statt. Mit dem «alten Tal» ist Professor Albert Heim gemeint, dem «Oeppi der Student» zu Zeiten auch als «Bibliothekar» aushalf (p. 476-480).

2 «Albert Heim. Leben und Forschung», von Marie Brockmann-Jerosch, Arnold und Helene Heim. Wepf, Basel: 1952. Unser Beitrag schöpft in vielen Punkten aus diesem Werk.

3 Zur Zeit ist immerhin eine Zürcher Dissertation in Arbeit: Marcel Gay, «Das Afrikabild Arnold Heims».

4. Zur Genealogie vgl. «Neue Deutsche Biographie, NDB», sowie R.Oehler in «Albert Heim. Leben und Forschung», p. 75-79.

5 R. J. Chorley im biographischen Abriß des «Dictionary of Scientific Biography, DSB».

6 «A. Heim. Leben ...» p. 17.

7 Vgl. J.Siebel, «Das Leben von Frau Dr. Marie Heim-Vögtlin, der ersten Schweizer Ärztin». Rascher, Zürich: 1919. Ferner: M. Lejeune-Jehle, «M. Heim-Vögtlin 1845-1916», in «Lebensbilder aus dem Aargau 1803-1953». Sauerländer, Aarau 1953, p.437-441; auch NDB.

8 «A. H. Leben ...» p. 12.

9 Chorley, l.c. (DSB).

10 In «Der Canton Glarus», von O. Heer, J. J.Blumer-Heer (u. a.). Huber, St. Gallen/Bern 1846, p. 68-70 («Gemälde der Schweiz» Band VII).

11 A.G. Milnes, «Albert Heim's general theory of natural rock deformation (1878)», in: «Geology (USA)» 7, 1979, p. 99-103.

12 Bezüglich A. Heims Reliefs vgl. Ed.Imhof, «Bildhauer der Berge». SAC, Bern, 1981, p. 128, 133 ff. u. a. a. O. ( = SA aus «Die Alpen. Zeitschrift des Schweizer Alpen-Clubs» 37, 1981, p. 103-166).

13 Vgl. «Oeppi der Student», p. 21-28.

14. Pars pro toto wieder Kübler, «Oeppi der Student», p.117, 163 u.a.a.O.

15 «A. H. Leben ...», p. 20 und 21.

16 Ebd.p.25.

17 Ebd.p.74.

18 Vierteljahrs. Naturforsch. Ges. Zürich 64 (1919), p.499-518 für 1866-1919; ebd. 74 (1929) Beibl. Nr.16 (5 S.) für 1919-1929; Verhandl. Schweiz. Naturforsch. Ges. 1937, p. 345-353 für 1929-1937. Vgl. Poggendorff, Band III-VII a.

19 Vgl. die in Anm.18 genannten Verzeichnisse; ferner Imhof, «Bildhauer der Berge», p. 153-159. 


\section{Summary*}

On the 31st of August fifty years will have elapsed since the distinguished Swiss geologist Albert Heim (1849-1937) departed from this life. When his biography was published in 1952 the strong personality of the imposing man of learning stood yet out clearly in the memory of the public, but to-day his name is presumably familiar only to the guild of professionals. Besides his achievements in the province of geology Heim's human qualities deserve wider consideration.

The article starts with an outline of Heim's career, the main stages of which were his origin, the years of study at the ETH Zurich, his succession to Professor Arnold Escher von der Linth's chair at ETH and Zurich University, his marriage with Marie Vögtlin, the first woman to practise as a doctor of medicine in Switzerland, academic tuition for more than the length of one generation (until 1911), and his later years.

To somewhat greater length the rich scientific bequest is being summarized: Heim's arduous path from his spectacular theory of the double fold in the Glarus Alps until the final acceptance of the nappe or decken theory, his standard works «Investigations into the mechanism of orogenesis» (1878), "Handbook of glaciology» (1885), «Geology of Switzerland» (1916ff.), his contributions to geological cartography, his remarkable terrain relievo models and Alpine panorama drawings, his ardent activities as a consultant and as a member of various committees, his renown as a dog-breeder, and his social commitment in favour of environmental protection, the equality of men and women and an addiction-free conduct of life. - Heim's voluminous literary bequest is deposited in the Central and ETH libraries in Zurich.

Frau Dr. S. Franks

Dr. Beat Glaus

ETH-Bibliothek

Rämistraße 101

CH-8092 Zürich

*Courtesy Dr. Heinz Lutstorf, ETH-Bibliothek. 
